\title{
All-Optical Membrane InP Switch on Silicon for Access Applications
}

\author{
O.Raz $^{(1)}$, M. Tassaert ${ }^{(2)}$, G. Roelkens ${ }^{(1,2)}$, H.J.S. Dorren ${ }^{(1)}$ \\ (1) Eindhoven University of Technology, Den Dolech 2, Eindhoven, The Netherlands \\ (2) INTEC, Ghent University-imec, Sint-Pietersnieuwstraat 41, 9000 Ghent, Belgium \\ Authore-mail address: o.raz@tue.nl
}

\begin{abstract}
Using an integrated membrane switch on SOI, optical clock distribution is achieved while all-optical switching of datapackets is maintained. Transmission through $25 \mathrm{~km}$ SMF is demonstrated with $1.5 \mathrm{~dB}$ penalty, limited by signal OSNR and pump extinction.

OCIS codes: (130.0130) Integrated optics; (130.4815) Optical switching devices; (060.6719) Switching, packet.
\end{abstract}

\section{Introduction}

The exponential increase in demand for bandwidth is forcing access networks to extend the amount of bandwidth they can support. Traditionally based on "static" allocation of time slots per user (or TDM) and passive optical splitters, these systems are struggling to keep up with the rise in line-rate as it cuts away link budget and therefore splitting ratio and network reach.

One possible solution is to replace passive splitters with active optical switches [1,2]. In these demonstrations, electronically controlled switches are used to choose between a simple passive splitting and a more advanced WDM based filtering. Since traditionally PON splitter boxes are located in cabinets where no electric power is present, power supply based on photo-voltaic cells was suggested. However also in these demonstrations, the emphasis was on allowing network reconfigurability rather than packet-based switching. In an attempt to implement a more dynamic packet switch, PLZT switches [3] (Lead Lanthanum Zirconate Titanate) were used. While switching speeds of $10 \mathrm{~ns}$ are possible, their insertion losses and power consumption are very high.

An alternative solution to passive splitting is the use of wavelength division multiplexing (WDM-PON) combined with passive splitters [4] to increase the number of subscribers and their share of the total network bandwidth while using low loss optical demultiplexers. This way some flexibility can be obtained in the allocation of bandwidth, but users linked to a certain wavelength output of the demultiplexer still have to compete with other users on the same branch for bandwidth through TDM, and physically re-wiring the network to double the number of wavelengths routed to a certain sub-network is not possible.

Previously we have introduced the membrane InP Switch (MIPS) as an all-optical switch with high extinction ratio and no pattern dependence. The MIPS was introduced as a single switching element which can be implemented in a broadcast-and-select architecture to create $1 \mathrm{xm}$ switches [5]. In this paper, we show that the unique absorption properties of the MIPS allow for transparent optical clock distribution simultaneously with high extinction ratio data packet switching. This combination can play a major role in future high-speed access networks. The distribution of a clock signal, allows for packet-based data transmission and detection without the need to recover the clock from the data packet and the high extinction ratio ensures low cross talk and rejection of undesirable packets. We validate the operation of the MIPS for such application by placing the switch $25 \mathrm{~km}$ downstream from the transmitter and measuring the bit-error-rate (BER) using a standard receiver and the transparently propagating clock. Measured receiver sensitivity penalty is kept to $1.5 \mathrm{~dB}$ mostly due to low OSNR and limited on/off ratio of the pump signal.

\section{Device fabrication and characteristics}

The InP membrane switch is made by adhesively bonding a thin $(<100 \mathrm{~nm})$ layer of epitaxially grown stack of InGaAs quantum wells and further post-processing of the membrane to leave a $2 \mu \mathrm{m}$ wide and $150 \mu \mathrm{m}$ long $\mathrm{InP}$ stripe on top of a silicon-on-insulator (SOI) waveguide circuit. In order to achieve broadband operation of the MIPS, previously used grating couplers were replaced by SU-8 inverted tapers, to expand the beam to better match with the mode field distribution of a lensed fiber and to achieve more broadband optical coupling to and from the chip. Light injected into the SOI waveguide was then transferred from the SOI to the InP switch structure by using a vertically positioned inverted taper with appropriate lengths $(18 \mu \mathrm{m}$ long $)$ in both the SOI and InP layers.

In Figure 1 an artist's impression of the MIPS layout is shown together with an SEM image of one such device. The InGaAs QWs are designed with a band gap of $1.58 \mu \mathrm{m}$. This means that light absorption is critically dependent on the wavelength, and since the membrane has such a small volume, very low optical power levels can render the InP structure transparent. In addition, since absorption is governed by the material band gap, optical signals at 
different wavelength will experience different absorption. In figure 2 we show the optical spectrum of the pump, data and clock signals at the input and output of the MIPS.

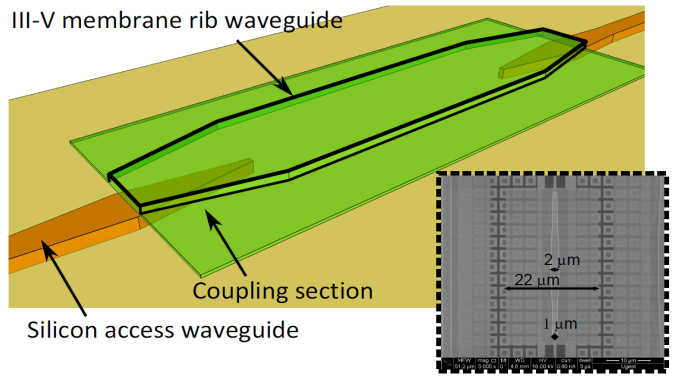

Fig. 1. Schematic view of the device. In the inset, a SEM image of a $50 \mu \mathrm{m}$ long device is shown.

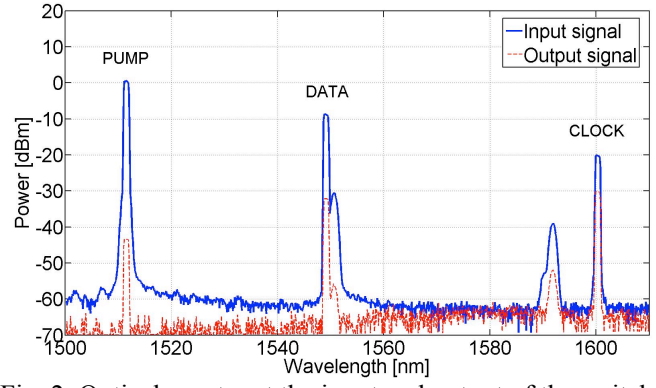

Fig. 2. Optical spectra at the input and output of the switch

The loss incurred by coupling light in and out of the silicon chip on which the membrane switch is integrated using lensed fibers is $10 \mathrm{~dB}$. However the III-V membrane will cause additional absorption for the pump, data and clock signals based on their wavelength. This leads to the following choice of wavelength: Broadcast clock signal at $1600 \mathrm{~nm}$, for minimal absorption; Data signal in the C-band with moderate extinction ratio (statically measured to be $15 \mathrm{~dB}$ ); Pump signal in the S-band where absorption is maximum for better utilization in bleaching the switch. As can be seen in the figure, the different signals indeed incur varying additional losses from $0 \mathrm{~dB}$ for the clock signal up to $34 \mathrm{~dB}$ for the pump signal.

\section{Experimental setup}

The experimental setup used for the demonstration is shown in figure 3. Three laser sources are used to generate the pump, data and clock signals. The data signal is comprised of packets of 1023 bits (PRBS 210-1 and a guard time of 50 bits (5 ns)). The generated packets are NRZ modulated at a bit rate of $10 \mathrm{~Gb} / \mathrm{s}$ on a laser with a wavelength of $1549.32 \mathrm{~nm}$ and combined through a cyclic Arrayed Waveguide Grating (AWG) with the clock signal which is modulated using the clock output of the data pattern generator with a wavelength of $1600.475 \mathrm{~nm}$ and a pump wavelength which is modulated by a second pattern generator (using the same clock) to make the required pump pulses at a wavelength of $1511.65 \mathrm{~nm}$. Packet length was chosen to be about $100 \mathrm{~ns}$ so that the AC coupled detector will not cause any AC coupling related eye closure in the error detector. This also led to the choice of the pattern length. It is important to mention that the switch itself does not inflict any pattern dependent penalty as we have previously reported [5].

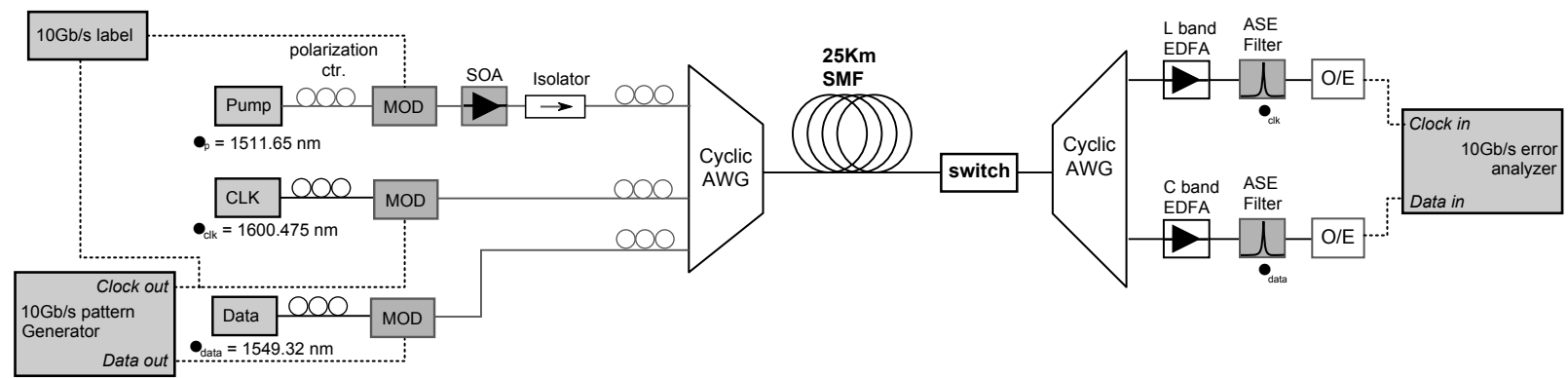

Fig. 3. Experimental setup

The pump signal is also amplified using a semiconductor optical amplifier to deliver a stronger pump signal. At the input to MIPS (after propagation through a $25 \mathrm{~km}$ of spooled single mode fiber) the pump, data and clock signals have powers of $0,-9$ and $-20 \mathrm{dBm}$ respectively.

Coming out of the MIPS, the signals are sent again through an identical AWG for demultiplexing before they are amplified and filtered. The relatively high fiber to fiber loss of $10 \mathrm{~dB}$, meant that both clock and data signals available after the AWG were weak, forcing the addition of two EDFA pre-amplifiers leading to relatively poor OSNR. Since $10 \mathrm{~Gb} / \mathrm{s}$ error free operation can also be achieved with power levels as low as $-25 \mathrm{dBm}$, improved SU8 inverted tapers [6] can drastically simplify the network and eliminate the need of the EDFAs at the ONU. After detection the obtained detected clock was filtered using a high-Q OC-192 RF filter and injected into the error analyzer together with the received data signal to analyze the performance of the link. 


\section{Results}

In figure 4, the obtained electrical signal at the output of the data receiver is presented. The pump signal is programmed for $105 \mathrm{~ns}$ on-time and $105 \mathrm{~ns}$ off-time. In the figure one can see the effect of the limited extinction ratio of the modulator used to turn the pump signal on and off. In a real application, seeing the very slow modulation speed needed ( $\sim 5-10 \mathrm{MHz})$, direct current modulation can be used to completely turn off the pump signal therefore improving the undesirable cross talk during the off state.

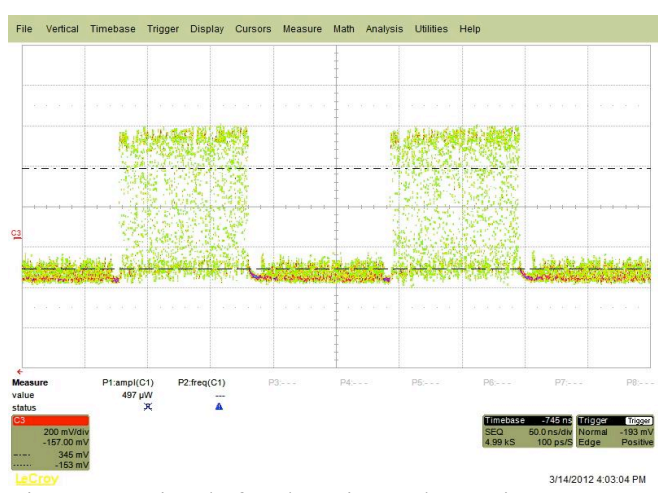

Fig. 4. Data signal after detection at the receiver

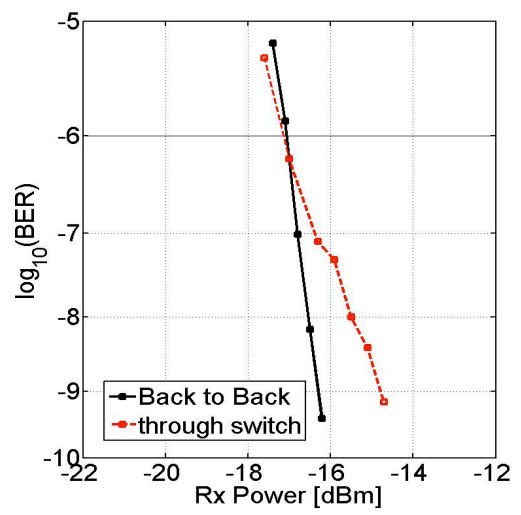

Fig. 5. BER Vs. Rx sensitivity performance comparison

In figure 5 we present the resulting BER performance of the link. At a bit error rate of $10^{-9}$ the signal traveling through the switch incurs an additional $1.5 \mathrm{~dB}$ power penalty compared to a back-to-back measurement. The measured performance takes into consideration the extra $3 \mathrm{~dB}$ peak-to-average power ratio of the partially transmitted data stream (see figure 4). The performance through the switch is limited by sig-spontaneous shot noise, while the back to back case is thermal noise limited. This in turn translates into different slopes for the two curves, and the different penalties at different BERs.

\section{Discussion and conclusions}

We have presented an optically controllable membrane InP switch (MIPS) which has a good extinction ratio and is transparent to longer wavelengths. We include the switch in a typical PON network down link with $25 \mathrm{~km}$ of fiber, and show that all-optical control of the switching element can be performed using a remote pump source (co-located with the downstream data transmitter) with a low receiver sensitivity penalty $(1.5 \mathrm{~dB})$ and simple $10 \mathrm{~Gb} / \mathrm{s}$ receiver circuits for the data and clock signals. The penalty is in part due to relatively high insertion loss of the unpackaged silicon-on-insulator chip and for the other part due to limited extinction ratio of the pump signal. We believe that by improving the losses of the chip and replacing the external modulator used to modulate the pump by direct current modulation we can eliminate the penalty all together. By implementing all the recommended improvements to the system, the power consumption of the switch can be minimized to the power needed to turn the MIPS on and off. We estimate that this power can be as low as several hundred microwatts, leading to energy consumption below $100 \mathrm{fJ}$ per bit (at $10 \mathrm{~Gb} / \mathrm{s})$.

In future work the MIPS will be implemented in a $1 \mathrm{xm}$ broadcast-and-select switch architecture [5]. To allow the optical clock distribution, the clock signal is placed in band with the data signal, such that it is broadcast to all nodes downstream.

\section{References}

[1] H. Ramanitra et al, "Scalable and multi-service passive optical access infrastructure using variable optical splitters," in OFCNFOEC 2006, IEEE, pp. 2391-2393.

[2] Jong Hoon Lee et al, "A remotely reconfigurable remote node for next-generation access networks," IEEE Phot. Tech. Lett. 20(11), pp. 915-917 (2008).

[3] Yutaro Hara et al, "A study of next generation metro-access hybrid scalable network by using PLZT ultra high speed optical wavelength selective switch," in 1st International Symposium on Access Spaces (ISAS) 2011, IEEE, pp. 1-6.

[4] Jim Theodoras et al, "100GbE - the Future of Ethernet," ADVA White paper (2010).

[5] M. Tassaert, et al, "Bias-free, low power and optically driven membrane InP switch on SOI for remotely configurable photonic packet switches,” Opt. Express 19(26), pp.B817-B824 (2011)

[6] M. Pu et al, "Ultra-low-loss inverted taper coupler for silicon-on-insulator ridge waveguide," Opt. commun. 283, pp. 3678-3682, 2010. 